\title{
CUA Guideline: The workup of azoospermic males
}

\author{
Keith Jarvi, MD, FRCSC; Kirk Lo, MD, FRCSC; Anthony Fischer, MD, FRCSC; John Grantmyre, MD, FRCSC; \\ Armand Zini, MD, FRCSC; Victor Chow, MD, FRCSC; Victor Mak, MD, FRCSC
}

A committee was established at the request of the CUA to determine guidelines for the investigation and management of azoospermia. Members of the committee, all of whom have special expertise in the investigation and management of male infertility, were chosen from different communities across Canada. The members represent different practices in different communities.

Can Urol Assoc J 2010;4(3):163-7

\section{Introduction}

Infertility or subfertility affects $15 \%$ of couples in Canada, with a male factor contributing to the fertility problem in close to $50 \%$ of these couples. Of the men presenting for fertility investigation, up to $20 \%$ are found to be azoospermic. These men can be categorized as having either:

1. pre-testicular azoospermia ( $2 \%$ of men with azoospermia, due to a hypothalamic or pituitary abnormality diagnosed with hypo-gonadotropic-hypogonadism),

2. testicular failure or non-obstructive azoospermia (49\% to $93 \%$, while the term testicular failure would seem to indicate a complete absence of spermatogenesis, actually men with testicular failure have either reduced spermatogenesis [hypospermatogenesis], maturation arrest at either an early or late stage of spermatogenesis or a complete failure of spermatogenesis noted with Sertoli-cell only syndrome), ${ }^{1-5}$

3. post-testicular obstruction or retrograde ejaculation (7\% to 51\%, normal spermatogenesis but obstructive azoospermia or retrograde ejaculation). ${ }^{1-5}$

A further group of men have a failure to ejaculate. These may be men with spinal cord injury, psychogenic failure to ejaculate or neurological damage (sympathetic nerve damage from a retroperitoneal lymph node dissection for example).

To understand the management of azoospermia, it is important to also understand the assisted reproductive technologies (ARTs) (e.g., in-vitro fertilization [IVF]). Since the 1970s, breakthroughs in the ARTs have allowed us to offer potentially successful treatments for up to $98 \%$ of couples with male factor infertility. ${ }^{6}$ These significant advances had little to do with techniques to improve the sperm quality but relied on ARTs. These programs used techniques to increase the number of mature eggs produced by the women by manipulating the hormonal environment in the women using exogenous hormones (ovulation induction) followed by:

1. timed insemination: timed to optimize the pregnancy rates: either through intercourse or intra-uterine insemination of the partners washed sperm; or

2. IVF: oocytes are retrieved from the ovaries then are either incubated with the sperm in a dish; or

3. intra-cytoplasmic sperm injection (ICSI): injecting the sperm directly into the cytoplasm of the oocyte.

All of the above techniques are widely used to treat couples with male factor infertility.

In the United States in 2003, 122872 IVF/ICSI cycles were performed and over 35785 IVF babies were delivered. ${ }^{6}$ Also in the United States in 2003, it was estimated that more than $1.0 \%$ of all newborns are IVF or ICSI babies. In Denmark, 5\% of newborns are IVF/ICSI babies.

Using ICSI, it is now possible to produce a pregnancy with any live sperm (moving or not), from either the semen or any site within the male reproductive tract. Even men with azoospermia can now be offered sperm retrieval with ICSI. Sperm could be retrieved from any site in the reproductive tract and used for ICSI. These are the men who previously had very limited chances to ever have biologically related children. Pregnancy rates of close to $50 \%$ per cycle of ICSI (women under 35 years old) are expected, with the pregnancy rates independent of the site of the origin of the sperm. ${ }^{6}$

\section{History and initial investigations for men with azoospermina}

After at least 2 semen analyses have confirmed azoospermia, men should be investigated with a history, physical examination and laboratory and imaging studies. The history should include information about:

1. the infertility history, such as duration of infertility, whether the infertility is primary or secondary, any treatments to date, libido and sexual activity;

2. the general health of the men, with particular emphasis on the presence of diabetes, respiratory issues; 
Jarvi et al.

\begin{tabular}{|c|c|c|c|}
\hline & Cystic fibrosis & Karyotype & Y-microdeletion \\
\hline $\begin{array}{l}\text { Absence or } \\
\text { obstruction of the } \\
\text { vas deferens, } \\
\text { epididymis or } \\
\text { ejaculatory ducts }\end{array}$ & $25-80 \%$ & & \\
\hline Testicular failure & & $14 \%$ & $1-30 \%$ \\
\hline
\end{tabular}

3. the history of proven or suspected genito-urinary infections:

4. the exposure to agents which might have an adverse impact on spermatogenesis, including but not limited to:

5. medical agents like hormone/steroid therapy, antibiotics (sulphasalazine), alpha-blockers, 5 alpha-reductase inhibitors, chemotherapeutic agents,

6. environmental factors like pesticides, excessive heat on the testicles;

7. recreational drugs (marijuana, excessive alcohol);

8. the surgery of the reproductive tract (hydrocelectomies, varicocelectomies etc); and

9. the history of any genetic abnormalities in the patient or his family.

If the man has had exposure to any of the above agents, the agents should be discontinued and the semen retested in 3 to 6 months. If the man has had a recent serious medical illness or injury or he has evidence of a recent reproductive tract infection, semen testing should be repeated at least 3 months following recovery from the illness.

Physical examination should include a thorough general examination with particular attention paid to the scrotal exam (size and consistency of the testis, presence and grade of varicoceles and palpable vas deferens). The initial testing will depend on these findings.

\section{Reduced semen volume}

If the semen volume is reduced $(<1.5 \mathrm{~mL})$ and documented on repeat testing, careful questioning should elicit whether this is an artifact (missed the container, difficulty providing specimen, etc.) or truly a low semen volume. Low semen volume could be due to:

1. absence/abnormalities of the vas deferens/seminal vesicles,

2. retrograde ejaculation, or

3. failure of emission.

Testing the post-ejaculate urine should help determine if there is retrograde ejaculation.

Occasionally, an alpha agonist (use pseudoephedrine or other just before the semen testing) will convert retro- grade into antegrade ejaculation. Diabetic men often have retrograde ejaculation or failure of emission.

Physical examination will help determine if the vas deferens is present in the scrotum and a transrectal ultrasound (TRUS) will determine if the seminal vesicles and vas deferens close to the prostate are normal. If absence of the vas deferens and/or the seminal vesicle is identified, the man has about an $80 \%$ chance to carry a genetic alteration associated with cystic fibrosis. ${ }^{7}$ Cystic fibrosis testing should be performed on all men with absence of the vas deferens/seminal vesicles (Grade A recommendation) (Fig. 1).

Obstruction of the ejaculatory duct is detected by TRUS and is usually accompanied by dilation of the seminal vesicles (typically $>1.5 \mathrm{~cm}$ ). Vasography is not required and should be discouraged for men with an ejaculatory duct obstruction (Level of evidence 3, Grade C Recommendation). If an ejaculatory duct obstruction is identified, the man has about a $25 \%$ chance to carry a genetic alteration associated with cystic fibrosis. ${ }^{7}$ Cystic fibrosis testing should be performed on all men with ejaculatory duct cysts.

\section{Differentiating the causes of normal volume azoospermia}

As mentioned above, the categories of the etiology of azoospermia are:

1. pre-testicular azoospermia ( $2 \%$ : hypothalamic or pituitary etiology)

2. testicular failure or non-obstructive azoospermia ( $49 \%$ to $93 \%$ )

3. post-testicular obstruction ( $7 \%$ to $51 \%$ : normal spermatogenesis but obstructive azoospermia).

The category of azoospermia can often be determined by the luteinizing hormone ( $\mathrm{LH})$ and follicle-stimulating hormone (FSH) levels. The diagnosis of pre-testicular azoospermia is relatively uncomplicated: $\mathrm{LH}$ and FSH levels will be low and the testosterone levels will be either low or normal. Men with elevated FSH and LH and small testis bilaterally have non-obstructive azoospermia. However, men with normal levels of FSH and LH could have either non-obstructive or obstructive azoospermia. ${ }^{8}$ Unfortunately, there is no noninvasive method to differentiate obstructive from non-obstructive azoospermia in this group of men. A testicular biopsy is usually required to provide a definitive diagnosis (Fig. 2).

There have been several recent publications about the use of biomarkers in the semen and serum to differentiate obstructive from non-obstructive azoospermia. ${ }^{8-20} \mathrm{~A}$ number of authors report on the use of inhibin $B$ serum levels to determine testicular function. ${ }^{8,11-14,16,17,21}$ While inhibin $B$ levels are generally lower in those men with more severe testicular dysfunction and is undetectable in those with a Sertoli cell only pattern on testis biopsy, inhibin B levels in men with maturation arrest or hyopospermatogenesis patterns on testis biopsies may be identical to those found in men with full sper- 


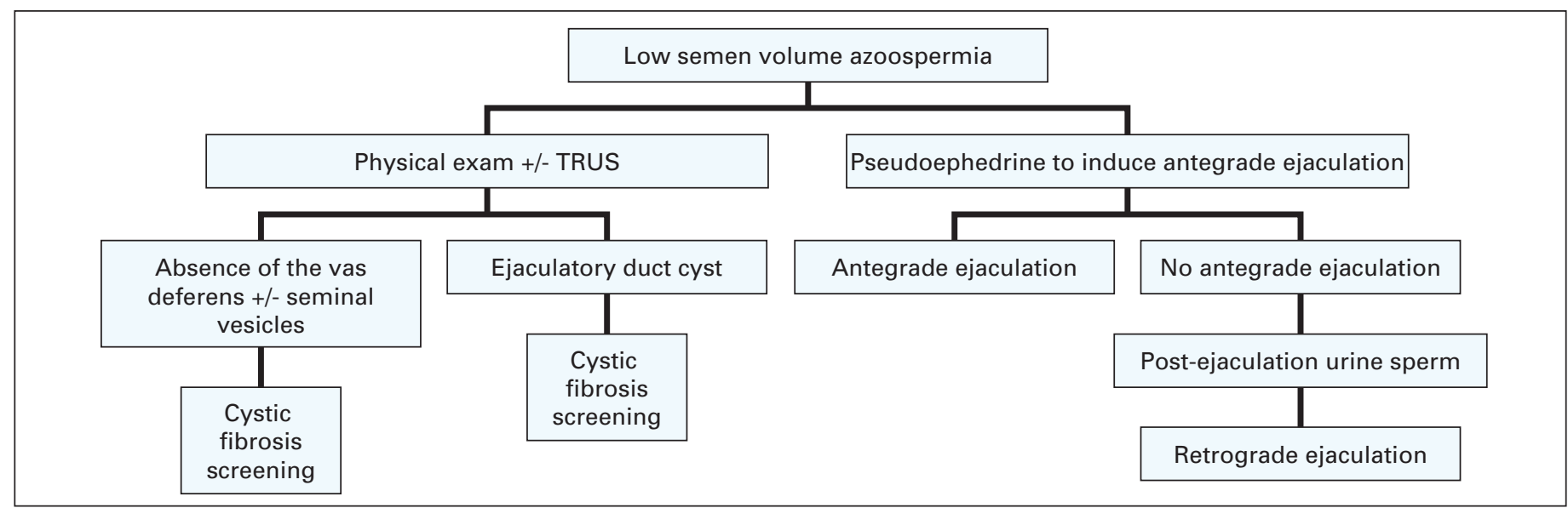

Fig. 1. Algorithm for the investigation of azoospermic men with low semen volume.

matogenesis. At present, serum inhibin B levels do not provide significant clinical benefit: with high $\mathrm{FSH}$ the inhibin $\mathrm{B}$ levels are generally low (both indicating testicular failure), while with normal FSH the inhibin B levels are generally normal (both indicating either obstructive or non-obstructive azoospermia) (Level of evidence 3, Grade C recommendation).

At present, for most men, there are no non-invasive methods to differentiate obstructive from non-obstructive azoospermia. As noted above, about $60 \%$ of men with azoospermia will require a testicular biopsy to provide a definitive diagnosis.

\section{Failure to ejaculate}

In men with a clear neurological cause (spinal cord injury, retroperitoneal lymph node surgery, etc.), no further investigations are required prior to treatment. Men with idiopathic failure to ejaculate (particularly those with a failure to orgasm) should be seen by a sex therapist.

\section{Genetic investigations for men with azoospermia}

All men with hypogonadotropic hypogonadism should be referred for genetics counselling as almost all of the congenital abnormalities of the hypothalamus are due to a genetic alteration.

All men with absence (absence of the vas deferens) or obstruction (epididymal or ejaculatory duct) of the reproductive tract ductal structures are at an elevated risk to carry a genetic alteration associated with cystic fibrosis. We recommend that not only the man but his partner should be offered cystic fibrosis testing in this situation. If a genetic alteration is identified, then genetic counselling is suggested (Level of evidence 2, Grade B recommendation).

All men with testicular failure should be offered karyotype and Y-micro-deletion testing then referred for genetics counselling if an abnormality is identified (Level of evidence 1, Grade A recommendation). ${ }^{22}$

\section{Management options for men with azoospermia}

Couples have many ways to achieve their goal of completing their family. The options of adoption, donor sperm and child-free living should always be discussed with the couple. The treatment options discussed below are those which allow a couple to have children biologically related to the man. These options depend on the diagnosis.

\section{Hypogonadotropic-hypogonadism or pre-testicular azoospermia}

This is best treated with the use of FSH/LH or GnRH analogues to stimulate spermatogenesis. In over $90 \%$ of the cases, spermatogenesis is induced and the men have ejaculated sperm. However, therapy may take more than 6 months to be effective.

\section{Retrograde ejaculation}

Use of pseudoephedrine or a similar alpha agonist may convert retrograde ejaculation into antegrade ejaculation. If this is not successful, it is often possible to retrieve sperm from the bladder (either using a post-ejaculatory voided or catherized urine specimen). This sperm could then be used for one of the ARTs. To optimize the sperm quality, it is often necessary to ask the men to alkalinize ( $\mathrm{pH}$ of $6.5-$ 8) the urine using standard medications.

\section{Obstructive azoospermia}

Obstructive azoospermia may be managed with:

1. Sperm retrieved from the reproductive tract (close to $100 \%$ chance of finding sperm) then the sperm is used in an ICSI program. The type of sperm retrieval used could be a percutaneous or an open microscopic aspiration of sperm from the epididymis or a percutaneous or open biopsy of the testis. Any of the types of retrievals listed above are acceptable. 
Jarvi et al.

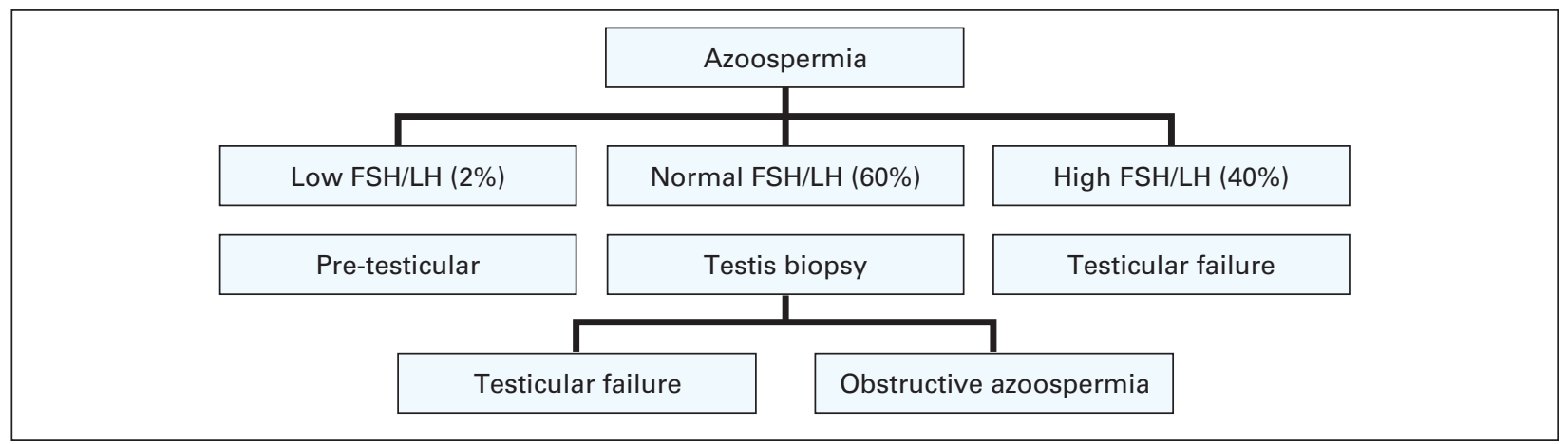

Fig. 2. Algorithm for differentiating the causes of normal semen volume azoospermia. $\mathrm{FSH}$ = follicle-stimulating hormone; $\mathrm{LH}$ = luteinizing hormone.

2. Bypass/repair of the obstructed area of the reproductive tract is possible in less than half of the men with obstructive azoospermia. ${ }^{22}$ The most common area of obstruction is within the epididymis. With the present microsurgical techniques, centres with expertise in performing vaso-epididymostomies report over $85 \%$ patency of the anastomosis (sperm in the ejaculate is the measure of patency) with over a 50\% spontaneous pregnancy rate. However, this is surgery requiring micro-surgical expertise and experience and should only be performed in centres with this kind of expertise. We recommend that all men be offered the option to cryo-bank sperm retrieved during the course of the operation in case the surgery is not successful (Level of evidence 3, Grade C recommendation).

3. Men with an ejaculatory duct obstruction may be candidates for a transurethral resection (TUR) ejaculatory duct. This is best performed using a TRUS guidance to allow the TUR to precisely unroof the ejaculatory duct cyst. It is important to warn men of the potential complications associated with a TUR of the prostate.

\section{Non-obstructive azoospermia}

Testicular sperm extraction may be used to identify sperm (reported success up to $75 \%$, mean $52 \%$ ) which could then be processed for use in an ICSI program. ${ }^{22-29}$ At present, the optimum way to identify these pockets of sperm is to perform an extensive, surgical dissection of the seminiferous tubules (a testicular sperm extraction) (Level of evidence 2, Grade B recommendation). Large sections of the seminiferous tubules of the testis are examined with an operating microscope. Those tubules which are larger in size are more likely to have spermatogenesis than smaller diameter tubules. The advantage of this technique over the regular random biopsy method is the ability to identify areas of the seminiferous tubules, which are more likely to contain sperm before the tissue is removed from the testicle. Using this technique the chance of finding sperm is higher than the older technique of taking random testicular biopsies alone (in one series $63 \%$ compared to $45 \%$ ), and while the procedure is laborious (surgical time may exceed 3 hours) the damage to the testicle is minimal due to the minimal amount of testis tissue eventually taken. ${ }^{28}$ ICSI pregnancy rates using sperm from a testicular sperm extraction program are reported to be between $19 \%$ to $50 \% .^{22,25-27,30}$ The testicular sperm extraction procedure should be offered to all men with nonobstructive azoospermia but should only be undertaken in a centre where ICSI is available.

\section{Failure to ejaculate}

Men with a neurological cause for a failure to ejaculate should be offered either vibro-stimulation or electroejaculation. Both of these procedures may cause autonomic dysreflexia in men with high spinal cord injuries. The semen specimen may be used for one of the ARTs. It is common that multiple (2 to 3 ) procedures several weeks apart may be needed to optimize the semen quality. These men may also have a concomitant obstruction in the epididymis, so occasionally sperm aspiration is required.

\section{When should the azoospermic man have a testis biopsy?}

As mentioned above, close to $60 \%$ of the men would need a testicular biopsy to document whether the azoospermia is post-testicular (normal spermatogenesis) or testicular (testicular failure with either Sertoli cell only syndrome, Maturation arrest pattern or hypospermatogenesis). However, a testis biopsy should only be offered to men in whom this diagnosis would alter management. As an example, we would discourage a man from having a testis biopsy if the couple is not interested in any of the potential management options that follow (e.g., things like sperm aspiration plus ICSI, vasoepididymostomy). If the couple is interested in considering the other fertility treatments mentioned above, then the biopsy could be performed by either of these 2 procedures.

The biopsy can be performed as a diagnostic procedure alone (either a percutaneous or an open biopsy are acceptable methods of testicular biopsies). The biopsy results then guide the next treatments. 
The biopsy can be the initial part of the larger fertility treatment. Once the biopsy results are available as a quick section, the surgery would then proceed with a reconstruction and/or sperm retrieval (if active spermatogenesis is detected) or a testicular sperm extraction (if a pattern of testicular failure is detected).

A bilateral diagnostic testicular biopsy is generally not required. If there is a discrepancy in testicular size, the larger of the 2 testes should be biopsied.

\section{What is the role of varicocelectomy in men with azoospermia?}

This remains controversial. There is some evidence that a small percentage of men with azoospermia due to testicular failure may benefit from treatment of a clinical varicocele. ${ }^{31}$ It is considered reasonable to offer men with clinical varicoceles and testicular failure a varicocele repair, but it is important to warn men that there is a low probability that this will result in any improvement in his semen parameters (Level of evidence 4, Grade D recommendation).

\section{What is the role of hormone therapy for men with azoospermia?}

Apart from the management of men with hypogonadotropic hypogonadism, the use of hormones to treat men with azoospermia should be discouraged. The use of androgens is contra-indicated (Level of evidence 1, Grade A recommendation).

Keith Jarvi, Director, Murray Koffler Urologic Wellness Centre, Head of Urology, Mount Sinai Hospital, Professor of Surgery, University of Toronto, Director, Male Infertility Program, Toronto, ON; Kirk Lo, Department of Surgery, Mount Sinai Hospital, Assistant Professor, University of Toronto, Toronto, ON; Anthony Fischer, Clinical Assistant Professor, McMaster University, Hamilton, ON; John Grantmyre, Department of Urology, Dalhousie University, Halifax, NS; Armand Zini, Associate Professor of Urology, McGill University, Head of the Division of Urology, St. Mary's Hospital, Montreal, QC; Victor Chow, Clinical Instructor, UBC, Vancouver, BC; Victor Mak, Urologist, Richmond Hill, ON

Competing Interests: None declared.

This paper has been peer-reviewed.

\section{References}

1. Fogle RH, Steiner AZ, Marshall FE, et al. Etiology of azoospermia in a large nonreferral inner-city popuIation. Fertil Steril 2006;86:197-9.

2. Jarow JP, Espeland MA, Lipshultz LI. Evaluation of the azoospermic patient. J Urol 1989;142:62-5.

3. Fedder J, Cruger D, Oestergaard B, et al. Etiology of azoospermia in 100 consecutive nonvasectomized men. Ferril Steril 2004;82:1463-5.

4. Wang C, So SY, Wong KK, et al. Chronic sinopulmonary disease in Chinese patients with obstructive azoospermia. J Androl 1987;8:225-9.
5. Matsumiya K, Namiki M, Takahara S, et al. Clinical study of azoospermia. Int J Androl 1994;17:140-2.

6. Assisted Reproductive Technology Success Rates. US Department of Health and Human Services, eds. 2005.

7. Mak V, Jarvi KA. The genetics of male infertility. J Urol 1996;156:1245-56; discussion 1256-7.

8. von Eckardstein $S$, Simoni $M$, Bergmann $M$, et al. Serum inhibin $B$ in combination with serum folliclestimulating hormone (FSH) is a more sensitive marker than serum FSH alone for impaired spermatogenesis in men, but cannot predict the presence of sperm in testicular tissue samples. I Clin Endocrinol Metab 1999;84:2496-501.

9. Chen DY, Wang JJ, Huang YF, et al. Relationship between lipocalin-type prostaglandin D synthase and alpha-glucosidase in azoospermia seminal plasma. Clin Chim Acta 2005;354:69-76.

10. Mitchell V, Brabant S, Koscinski I, et al. Proacrosin, an acrosomal marker for the detection of spermatogenic cells in ejaculates from azoospermic men [in French]. Gynecol Obstet Fertil 2004;32:779-84.

11. Bordallo MA, Guimarães MM, Pessoa $\mathrm{CH}$, et al. Decreased serum inhibin B/FSH ratio as a marker of Sertoli cell function in male survivors after chemotherapy in childhood and adolescence. J Pediatr Endocrinol Metab 2004; 17:879-87.

12. Frydelund-Larsen L, Krausz C, Leffers $\mathrm{H}$, et al. Inhibin B: a marker for the functional state of the seminiferous epithelium in patients with azoospermia factor C microdeletions. J Clin Endocrinol Metab 2002;87:5618-24.

13. Hipler UC, Hochheim B, Knoll B, et al. Serum inhibin B as a marker for spermatogenesis. Arch Androl 2001;46: 217-22

14. Brugo-Olmedo S, De Vincentiis S, Calamera IC, et al. Serum inhibin B may be a reliable marker of the presence of testicular spermatozoa in patients with nonobstructive azoospermia. Fertil Steril 2001;76:1124-9.

15. Zöpfgen A, Priem F, Sudhoff $F$, et al. Relationship between semen quality and the seminal plasma components carnitine, alpha-glucosidase, fructose, citrate and granulocyte elastase in infertile men compared with a normal population. Hum Reprod 2000;15:840-5.

16. Foresta $C$, Bettella A, Petraglia $F$, et al. Inhibin B levels in azoospermic subjects with cytologically characterized testicular pathology. Clin Endocinol (Oxf) 1999:50:695-701.

17. Foppiani L, Schlatt S, Simoni M, et al. Inhibin B is a more sensitive marker of spermatogenetic damage than FSH in the irradiated non-human primate model. J Endocrinol 1999;162:393-400.

18. Diamandis EP, Arnett WP, Foussias $G$, et al. Seminal plasma biochemical markers and their association with semen analysis findings. Urology 1999;53:596-603.

19. Fujisawa $M$, Tanaka $H$, Tatsumi N, et al. Telomerase activity in the testis of infertile patients with selected causes. Hum Reprod 1998;13:1476-9.

20. Kret $B$, Milad $M$, Jeyendran RS. New discriminatory level for glucosidase activity to diagnose epididymal obstruction or dysfunction. Arch Androl 1995;35:29-33.

21. Hu YA, Huang YF. A serum marker of spermatogenesis-inhibin B [in Chinese]. Zhonghua Nan Ke Xue 2002;8:57-60.

22. Schlegel PN. Causes of azoospermia and their management. Reprod Ferril Dev 2004;16:561-72.

23. Kim ED, Gilbaugh JH 3rd, Patel VR, et al. Testis biopsies frequently demonstrate sperm in men with azoospermia and significantly elevated follicle-stimulating hormone levels. J Urol 1997;157:144-6.

24. Gil Salom M. Spermatic recovery techniques for intracytoplasmic spermatozoid injection (ICSI) in male infertility [in Spanish]. Arch Esp Urol 2004:57:1035-46.

25. Schiff JD, Palermo GD, Veeck LL, et al. Success of testicular sperm extraction [corrected] and intracytoplasmic sperm iniection in men with Klinefelter syndrome. I Clin Endocrinol Metab 2005;90:6263-7.

26. Raman JD, Schlegel PN. Testicular sperm extraction with intracytoplasmic sperm injection is successful for the treatment of nonobstructive azoospermia associated with cryptorchidism. J Urol 2003;170:1287-90.

27. Chan PT, Palermo GD, Veeck $L$, et al. Testicular sperm extraction combined with intracytoplasmic sperm injection in the treatment of men with persistent azoospermia postchemotherapy. Cancer 2001;92:1632-7.

28. Schlegel PN. Testicular sperm extraction: microdissection improves sperm yield with minimal tissue excision. Hum Reprod 1999;14:131-5.

29. Schlegel PN, Palermo GD, Goldstein $M$, et al. Testicular sperm extraction with intracytoplasmic sperm injection for nonobstructive azoospermia. Urology 1997;49:435-40.

30. Colpi GM, Piediferro G, Nerva F, et al. Sperm retrieval for intra-cytoplasmic sperm injection in nonobstructive azoospermia. Minerva Urol Nefrol 2005;57:99-107.

31. Schlegel PN, Kaufmann J. Role of varicocelectomy in men with nonobstructive azoospermia. Fertil Steril 2004;81:1585-8

Correspondence: Dr. Keith Jarvi, Murray Koffler Urologic Wellness Centre, Mount Sinai Hospital, Joseph and Wolf Lebovic Health Complex, 60 Murray St., 6th Floor, Box 19, Toronto, ON M5T 3L9; fax: 416-586-4776; kjarvi@mtsinai.on.ca 